may be strong and of short range, they will not seriously modify the state, since all collisions with small momentum transfers are forbidden. Unfortunately, such an approach, using interactions containing sufficient exchange forces to produce satura. tion, gives too little binding-energy. The source of this discrepancy appears to be the fact that nuclear saturation arises only partly from exchange forces, another cause being the infinitely repulsive core in the nuclear interaction. The presence of this core means that shell-model wave-functions cannot possibly be correct when any two nucleons are separated by just the range of the core. Nevertheless, since the range of the core is considerably smaller than the mean inter-nucleon spacing, they are expected to be correct otherwise. Mathematical techniques have been developed for taking account of the effects of the cores in the wave-functions, and it is believed that one has, in principle, a reliable scheme for computing nuclear properties, especially binding-energies, using realistic nuclear forces. The main trouble, in practice, is the great labour involved in calculation. This reduces to reasonable magnitude only when considering large hypothetical systems in which surface effects are negligible. An important by product of such calculations is the estimation of the nuclear potential, $V$, as a function of the momentum of a nucleon. This emerges in an iteration procedure in which one assumes values for $V$, then calculates new values and continues until convergence is obtained. It can be identified with the real part of the potential of the optical model, and so the calculations predict this quantity as well as the nuclear binding energies.

The meeting ended after the speakers answered a few questions from the audience. A. M. LANE

\title{
BIOCHEMISTRY OF GLUTATHIONE
}

$\mathrm{T}$ HE Biochemical Society arranged a symposium on glutathione, organized by Dr. E. M. Crook and held on February 15 at the Senate House, University of London. As indicated by Prof. R. H. S. Thompson's opening remarks, the subject is attractive in bringing together a wide range of disciplines: purely chemical and analytical studies, plant and animal biochemistry, and pharmacology, toxicology and general biology.

The metabolism of glutathione in plants is notable, as described by Dr. L. W. Mapson (Low Temperature Research Station, Cambridge), for the well-defined. systems which catalyse both oxidation and reduction at its sulphide group. Glutathione reductase has been found in most organisms in which it has been sought. More characteristic of plants than animals is dehydroascorbic acid reductese, which catalyses the interaction of reduced glutathione with the oxidized form of ascorbic acid. The reduced ascorbic acid formed can be oxidized by established routes, and about a quarter or a half the oxygen uptake of preparations of germinating pea cotyledons may proceed by this route, providing an example of the respiratory function envisaged for glutathione by Hopkins and others. However, attempts to demonstrate the generation of high-energy bonds during these changes have so far been unsuccessful.

\section{Glutathione in Animal Tissues}

In animal tissues there appears relatively little scope for this route of oxidation; but oxidation by purely chemical routes, for example, by exchange with other disulphides, can readily occur. This and other aspects of the chemistry of glutathione were surveyed by Dr. F. A. Isherwood (Low Temperature Research Station, Cambridge); thus transacylation also occurs spontaneously between the thiol group of glutathione and S-acyl compounds. Oxidation and mercaptide formation at the glutathione thiol group form the basis of two analytical methods for glutathione described in detail by Dr. C. G. Thomson (Institute of Psychiatry, London), who compared results with these methods with those obtained using glyoxalase. A considerable measure of agre日- ment was found when these techniques were applied to blood and to cerebral tissues. Each tissue, however, presents its own problems in fixation, extraction, and the presence of substances likely to interfere in assay, as was evident also from discussion.

Reviewing glutathione in animal tissues generally, Dr. P. C. Jocelyn (University of Edinburgh) described recent studies of its synthesis. Synthesis proceeds through $\gamma$-glutamylcysteine and is catalysed by two distinet synthetases in tissues including erythrocytes and liver. In the liver, the synthesis appears to be affected by growth hormone and adrenaline; the extent to which vitamin $B_{12}$ is involved in the maintenance was also appraised. Although glutathione has been suggested as a substrate in the further synthesis of proteins, experiments on incorporation of amino-acids into ovalbumin, and other lines of evidence, have failed to support the idea.

In neural tissues, Prof. H. McIlwain (Institute of Psychiatry, University of London) described the determination of glutathione in the brain fixed rapidly in situ, and its maintenance during in vitro experiments. Assimilation, synthesis, and breakdown of glutathione in cerebral tissues proceed at relatively slow rates, as also does its acylation. Deacylation is more rapid, especially of an acetylglycyl glutathione. Enzyme catalysis of the oxidation of glutathione has not been found, but the tissue possesses a very active glutathione reductase which forms the main route of oxidation of reduced triphosphopyridine nucleotide. This system operates at nearly maximal rate at the low concentrations of oxidized glutathione and of reduced triphosphopyridine nucleotide which are native to the tissue. Indeed, its operation would appear to be the main factor in securing the great preponderance of the reduced form of glutathione normally found in vivo, and to be necessary if glutethione is to perform the role so often ascribed to it, of maintaining thiol enzymes and coenzymes in their active, reduced, forms. Participation of glutathione in cerebral carbohydrate metabolism is well established, and change in level of blood glutathione has been reported to be associated with mental disease. Administration of large quantities of glutathione to 
man with the intention of altering mental status has displeyed the relatively small central effects produced in this way, in distinction to the marked effects of other thiols on the nervous system. Blood constituents are, however, altered by the administration of such quantities.

Analogues of glutathione have been found to occur naturally in the lens of the eye. Their isolation was described by Dr. S. G. Waley (Nuffield Laboratory of Ophthalmology, Oxford); they differ from gluta. thione at its cysteinyl residue, ophthalmic acid being $\gamma$-glutamyl- $\alpha$-amino-n-butyrylglycine, and norophthalmic acid the alanyl derivative. They appear to be produced in the lens by the system which synthesizes glutathione, for competition can occur when substrates for more than one product are provided. Glutathion $\Theta$ synthesis is affected also by the X-irradiation which induces cataract ; after exposure, the glutathione concentration in the lens falls, but causal relationship between this change and the cataract is still under investigation. A further intriguing product isolated from the lens is S-sulphoglutathione, which can be formed from sulphite and oxidized glutathione.

\section{Radiation Damage; Glyoxalase}

In reviewing relationships between thiols and radiation damage generally, Dr. D. B. Hope (M.R.C. Radiobiological Research Unit, Harwell) described the inactivation of thiol enzymes brought about in vitro by irradiation. This has not been observed in vivo, so the enzymes involved are in some way protected, and attempts have been made to enhance the normal protection by administered substances. Glutathione to a small extent protects mice from the lethal effects of $\mathrm{X}$-irradiation, but cysteamine, dithiocarbamates, and other compounds are more effective; they have in common the property of lowering the body temperature of animals.

Interesting points regarding glyoxalase came from several contributors. This is the system converting methylglyoxal to lactic acid, in which coenzyme status for glutathione was first established, and which once appeared to constitute the main route of glycoly sis in many organisms. Its displacement from this role was exemplified in cerebral tissues : lactic acid formation from methylglyoxal, though rapid, is inadequate to account for the high rates at which lactic acid was afterwards found to be produced from glucose. That the glyoxalase system comprises two enzymes, adumbrated by F. G. Hopkins in his posthumous paper with $\mathrm{E}$. J. Morgan, is generally agreed. Perhaps surprisingly, the immediate sub. strate for glyoxalase I is still debated, Dr. Waley's evidence favouring the hemimercaptal between glutathione and methylglyoxal. In this system in the lens, ophthalmic acid acts as a competitive inhibitor. Glyoxalase I yields S-lactoylglutathione, the substrate of glyoxalase II. However, a preparation from cerebral tissues is reported to hydrolyse at least one other substrate more rapidly than glyoxalase intermediate: this is S-acetylglycylglutathione. Hydrolytic enzymes have also been found in mouse liver which are specific to S-acyl derivatives of glutathione.

Concluding remarks by Dr. C. P. Stewart (University of Edinburgh) emphasized these and other aspects of the biochemistry of glutathione in which further investigation is still required. $\mathrm{He}$ indicated how studies of radiation damage, and the $S$-sulphoglutathione from the lens, offer hints on compounds intermediate in the conversion of -SH to - S.S glutathione, a process likely to involve two stages.

\title{
NATIONAL OCEANOGRAPHIC COUNCIL
}

\author{
ANNUAL REPORT 1956-57
}

\begin{abstract}
$\mathrm{T}$ HE report of the National Oceanographic Council for 1956-57 (pp. $v+34$. Cambridge: At the University Press, 1957. 5s. net) describes the work carried out by the National Institute of Oceanography during the year ending March 31, 1957. The activities cover the two main fields of marine physics and marine biology, and follow the broad objectives set in previous years.

In marine physics, the technique of determining deep currents by tracking a neutrally buoyant float, drifting freely at a predetermined depth, has been exploited further. The most significant piece of work was the discovery of a southerly-flowing deep current, at depths greater than $2,500 \mathrm{~m}$., beneath the Gulf Stream. This was achieved in the course of a joint expedition by R.R.S. Discovery $I I$, and the U.S. research vessel Atlantis to an area about 150 miles off the coast of South Carolina. Other measurements of deep currents were made during the year in the Farroe-Shetland Channel, off the Sogne Fjord, and in a region north of Madeira.

Waves and swell have been one of the Institute's main interests since its inception, and during the past year or two increasing attention has been given to their effects on the rolling, pitching and heaving of ships. The work on this subject has attracted much
\end{abstract}

interest among scientists and engineers concerned with ship motion, in Great Britain and in the United States. A series of theoretical papers has also been published, treating the statistical properties of waves as those of a random, moving surface. Other studies of waves include their relation to microseisms and the relation of both to the movements of storms generating them. Turning to waves on a longer time-scale, basic research with a bearing on storm surges has been continued, both by mathematical models and by electrical analogue techniques.

The productivity of oceanic waters is one of the Institute's major objectives in marine biology, and advances have been made in instrumental and operational techniques for this purpose. An example of this is provided by work on the quantitative sampling of zooplankton from water layers at various depths. Research at sea on organic productivity included three weeks observations in one position, 600 miles west of Portugal, during the autumn of 1956. Repeated measurements were made from Discovery II of the light energy, rate of photosynthesis and the standing crop of phytoplankton and zooplankton.

Another broad field of investigation concerns the oceanic distribution of marine organisms. Compre- 